\title{
Poly-Dedekind sums associated with poly-Bernoulli functions
}

\author{
Yuankui Ma', Dae San Kim², Hyunseok Lee ${ }^{3}$ and Taekyun Kim,3*
}

\section{"Correspondence:}

kwangwoonmath@hanmail.net 'School of Science, Xi'an

Technological University, Xi'an, 710021, Shaanxi, P.R. China ${ }^{3}$ Department of Mathematics, Kwangwoon University, Seoul 139-701, Republic of Korea

Full list of author information is available at the end of the article

\begin{abstract}
Apostol considered generalized Dedekind sums by replacing the first Bernoulli function appearing in Dedekind sums by any Bernoulli functions and derived a reciprocity relation for them. Recently, poly-Dedekind sums were introduced by replacing the first Bernoulli function appearing in Dedekind sums by any type 2 poly-Bernoulli functions of arbitrary indices and were shown to satisfy a reciprocity relation. In this paper, we consider other poly-Dedekind sums that are obtained by replacing the first Bernoulli function appearing in Dedekind sums by any poly-Bernoulli functions of arbitrary indices. We derive a reciprocity relation for these poly-Dedekind sums.
\end{abstract}

MSC: 11F20; 11B68; 11B83

Keywords: Poly-Dedekind sum; Polylogarithmic function; Poly-Bernoulli polynomial

\section{Introduction}

The sawtooth function, denoted by $((x))$, is defined by

$$
((x))=\left\{\begin{array}{ll}
x-[x]-\frac{1}{2}, & \text { if } x \notin \mathbb{Z}, \\
0, & \text { if } x \in \mathbb{Z},
\end{array} \quad \text { (see }[1-5]\right),
$$

where $[x]$ denotes the greatest integer function not exceeding $x$.

The Dedekind sums are defined by

$$
\begin{aligned}
S(h, m) & =\sum_{\mu=1}^{m-1}\left(\left(\frac{\mu}{m}\right)\right)\left(\left(\frac{h \mu}{m}\right)\right) \\
& =\sum_{\mu=1}^{m-1}\left(\frac{\mu}{m}-\frac{1}{2}\right)\left(\left(\frac{h \mu}{m}\right)\right) \\
& =\sum_{\mu=1}^{m-1} \frac{\mu}{m}\left(\left(\frac{h \mu}{m}\right)\right),
\end{aligned}
$$

\section{Springer}

where $h$ is any integer and $m$ is a positive integer (see $[9-11,17,19,20])$.

(c) The Author(s) 2020. This article is licensed under a Creative Commons Attribution 4.0 International License, which permits use sharing, adaptation, distribution and reproduction in any medium or format, as long as you give appropriate credit to the original author(s) and the source, provide a link to the Creative Commons licence, and indicate if changes were made. The images or other third party material in this article are included in the article's Creative Commons licence, unless indicated otherwise in a credit line to the material. If material is not included in the article's Creative Commons licence and your intended use is not permitted by statutory regulation or exceeds the permitted use, you will need to obtain permission directly from the copyright holder. To view a copy of this licence, visit http://creativecommons.org/licenses/by/4.0/. 
It is well known that the Bernoulli polynomials are defined by

$$
\frac{t}{e^{t}-1} e^{x t}=\sum_{n=0}^{\infty} B_{n}(x) \frac{t^{n}}{n !} \quad(|t|<2 \pi),(\text { see }[1-13,17,19,20]) .
$$

When $x=0, B_{n}=B_{n}(0),(n \geq 0)$ are called the Bernoulli numbers.

From (3), we note that

$$
B_{n}(x)=\sum_{l=0}^{n}\left(\begin{array}{l}
n \\
l
\end{array}\right) B_{n-l} x^{l} \quad(n \geq 0),(\text { see }[7-13])
$$

By (3), we easily get

$$
\sum_{l=0}^{n-1} l^{m}=\frac{1}{m+1}\left(B_{m+1}(n)-B_{m+1}\right), \quad(n \in \mathbb{N}, m \geq 0),(\text { see [13]), }
$$

and

$$
d^{n-1} \sum_{l=0}^{d-1} B_{n}\left(\frac{x+i}{d}\right)=B_{n}(x), \quad(n \geq 0, d \in \mathbb{N}),(\text { see }[10,13])
$$

The modified Hardy's polyexponential function of index $k$ is defined by

$$
\operatorname{Ei}_{k}(x)=\sum_{n=1}^{\infty} \frac{x^{n}}{n^{k}(n-1) !} \quad(k \in \mathbb{Z}),(\text { see }[7]) .
$$

Note that $\operatorname{Ei}_{1}(x)=e^{x}-1$.

Recently, the type 2 poly-Bernoulli polynomials of index $k$ are defined by

$$
\frac{\mathrm{Ei}_{k}(\log (1+t))}{e^{t}-1} e^{x t}=\sum_{n=0}^{\infty} B_{n}^{(k)}(x) \frac{t^{n}}{n !} \quad(k \in \mathbb{Z})
$$

When $x=0, B_{n}^{(k)}=B_{n}^{(k)}(0),(n \geq 0)$ are called the type 2 poly-Bernoulli numbers of index $k$. Note that $B_{n}^{(1)}(x)=B_{n}(x),(n \geq 0)$.

It is well known that the polylogarithmic function of index $k$ is defined by

$$
\operatorname{Li}_{k}(x)=\sum_{n=1}^{\infty} \frac{x^{n}}{n^{k}}, \quad(k \in \mathbb{Z}),|x|<1,(\text { see }[6,9,12]) .
$$

Note that $\operatorname{Li}_{1}(x)=-\log (1-x)$.

In $[6,7,12]$, the poly-Bernoulli polynomials of index $k$ are defined by the generating function

$$
\frac{\mathrm{Li}_{k}\left(1-e^{-t}\right)}{e^{t}-1} e^{x t}=\sum_{n=0}^{\infty} \beta_{n}^{(k)}(x) \frac{t^{n}}{n !} .
$$

When $x=0, \beta_{n}^{(k)}=\beta_{n}^{(k)}(0)$ are called the poly-Bernoulli numbers of index $k$. 
From (10), we note that

$$
\sum_{l=0}^{n}\left(\begin{array}{l}
n \\
l
\end{array}\right) \beta_{n-l}^{(k)} x^{l}=\beta_{n}^{(k)}(x), \quad(n \geq 0),(\text { see }[6,7,12]) .
$$

The fractional part of $x$ is defined by

$$
\langle x\rangle=x-[x]
$$

The Bernoulli functions are defined by

$$
\bar{B}_{n}(x)=B_{n}(\langle x\rangle), \quad(n \geq 0),(\text { see }[1,2]) .
$$

From (2), we have

$$
\begin{aligned}
S(h, m) & =\sum_{\mu=1}^{m-1} \frac{\mu}{m}\left(\frac{h \mu}{m}-\left[\frac{h \mu}{m}\right]-\frac{1}{2}\right) \\
& =\sum_{\mu=1}^{m-1}\left(\frac{\mu}{m}-\frac{1}{2}\right)\left(\frac{h \mu}{m}-\left[\frac{h \mu}{m}\right]-\frac{1}{2}\right) \\
& =\sum_{\mu=1}^{m-1} \bar{B}_{1}\left(\frac{\mu}{m}\right) \bar{B}_{1}\left(\frac{h \mu}{m}\right),
\end{aligned}
$$

where $h, m$ are relatively prime positive integers.

Apostol considered the generalized Dedekind sums, which are given by

$$
S_{p}(h, m)=\sum_{\mu=1}^{m-1} \frac{\mu}{m} \bar{B}_{p}\left(\frac{h \mu}{m}\right)
$$

and showed in $[1,2]$ that they satisfy the reciprocity relation

$$
(p+1)\left(h m^{p} S_{p}(h, m)+m h^{p} S_{p}(m, h)\right)=p B_{p+1}+\sum_{s=0}^{p+1}\left(\begin{array}{c}
p+1 \\
s
\end{array}\right)(-1)^{s} B_{s} B_{p+1-s} h^{s} m^{p+1-s}
$$

As one generalization of Apostol's generalized Dedekind sums, the poly-Dedekind sums associated with the type 2 poly-Bernoulli functions of index $k$

$$
S_{P}^{(k)}(h, m)=\sum_{\mu=1}^{m-1} \frac{\mu}{m} \bar{B}_{p}^{(k)}\left(\frac{h \mu}{m}\right)
$$

were recently introduced (see [13]) and, among other things, a reciprocity relation for them was derived.

In this paper, as another generalization of Apostol's generalized Dedekind sums, we consider the poly-Dedekind sums defined by

$$
T_{p}^{(k)}(h, m)=\sum_{\mu=1}^{m-1} \frac{\mu}{m} \bar{\beta}_{p}^{(k)}\left(\frac{h \mu}{m}\right)
$$


where $\bar{\beta}_{p}^{(k)}(x)=\beta_{p}^{(k)}(\langle x\rangle)$ are the poly-Bernoulli functions of index $k$ (see (10)). Note here that $T_{p}^{(1)}(h, m)=S_{p}(h, m)$. We show the following reciprocity relation for the polyDedekind sums given by (see Theorem 7 )

$$
\begin{aligned}
& h m^{p} T_{p}^{(k)}(h, m)+m h^{p} T_{p}^{(k)}(m, h) \\
&= \sum_{\mu=0}^{m-1} \sum_{j=0}^{p} \sum_{\nu=0}^{h-1} \sum_{l=1}^{p-j+1} \frac{(m h)^{j-1} l ! S_{2}(p-j+1, l)}{(p-j+1) l^{k}} \\
& \quad \times\left(\begin{array}{c}
p \\
j
\end{array}\right)(-1)^{p-j+1-l}\left((\mu h) m^{p-j}+(m v) h^{p-j}\right) \bar{B}_{j}\left(\frac{v}{h}+\frac{\mu}{m}\right) .
\end{aligned}
$$

For $k=1$, this reciprocity relation for the poly-Dedekind sums reduces to that for Apostol's generalized Dedekind sums given by (see Corollary 8 )

$$
\begin{aligned}
& h m^{p} S_{p}(h, m)+m h^{p} S_{p}(m, h) \\
& \quad=\sum_{\mu=0}^{m-1} \sum_{\nu=0}^{h-1}(m h)^{p-1}(\mu h+m v) \bar{B}_{p}\left(\frac{v}{h}+\frac{\mu}{m}\right) .
\end{aligned}
$$

We recommend the readers to look at the articles $[15,16,18,21]$ and the more recent one [14], which are related to the present paper. In Sect. 2, we derive various facts about the poly-Bernoulli polynomials that will be needed in the next section. In Sect. 3, we define the poly-Dedekind sums associated with the poly-Bernoulli functions and demonstrate a reciprocity relation for them.

\section{Poly-Dedekind sums associated with poly-Bernoulli functions}

Let $n$ be a nonnegative integer. Then the Stirling numbers of the second kind are defined by

$$
x^{n}=\sum_{k=0}^{n} S_{2}(n, k)(x)_{k}, \quad(n \geq 0),(\text { see }[1-14,17,19])
$$

where $(x)_{0}=1,(x)_{n}=x(x-1) \cdots(x-n+1),(n \geq 1)$.

From (9) and (10), we note that

$$
\frac{\mathrm{Li}_{k}\left(1-e^{-t}\right)}{e^{t}-1}=\sum_{n=0}^{\infty} \beta_{n}^{(k)} \frac{t^{n}}{n !}
$$

Thus, by (15), we get

$$
\begin{aligned}
\operatorname{Li}_{k}\left(1-e^{-t}\right) & =\left(\sum_{l=0}^{\infty} \beta_{l}^{(k)} \frac{t^{l}}{l !}\right)\left(e^{t}-1\right) \\
& =\sum_{n=0}^{\infty}\left(\beta_{n}^{(k)}(1)-\beta_{n}^{(k)}\right) \frac{t^{n}}{n !} .
\end{aligned}
$$


On the other hand,

$$
\begin{aligned}
\operatorname{Li}_{k}\left(1-e^{-t}\right) & =\sum_{m=1}^{\infty} \frac{1}{m^{k}}\left(1-e^{-t}\right)^{m}=\sum_{m=1}^{\infty} \frac{(-1)^{m} m !}{m^{k}} \frac{1}{m !}\left(e^{-t}-1\right)^{m} \\
& =\sum_{m=1}^{\infty} \frac{(-1)^{m} m !}{m^{k}} \sum_{n=m}^{\infty} S_{2}(n, m)(-1)^{n} \frac{t^{n}}{n !} \\
& =\sum_{m=1}^{\infty}\left(\sum_{m=1}^{n} \frac{(-1)^{n-m} m !}{m^{k}} S_{2}(n, m)\right) \frac{t^{n}}{n !} .
\end{aligned}
$$

Therefore, by (16) and (17), we obtain the following theorem.

Theorem 1 For $n \in \mathbb{N}$, we have

$$
\beta_{n}^{(k)}(1)-\beta_{n}^{(k)}=\sum_{m=1}^{n} \frac{(-1)^{n-m} m !}{m^{k}} S_{2}(n, m)
$$

From Theorem 1, we note that

$$
\beta_{0}^{(k)}=1, \quad \beta_{1}^{(k)}=-1+\frac{1}{2^{k}}, \quad \beta_{2}^{(k)}=1-\frac{3}{2^{k}}+\frac{2}{3^{k}}, \ldots
$$

Taking $k=1$ in Theorem 1 gives us the following corollary.

Corollary 2 For $n \in \mathbb{N}$, we have

$$
\sum_{m=1}^{n}(-1)^{n-m}(m-1) ! S_{2}(n, m)=\delta_{n, 1}
$$

where $\delta_{n, k}$ is the Kronecker symbol.

The three identities in the following lemma can be shown just as in Theorem 3, Corollary 4, and Theorem 5 of [13], and hence their proofs are left to the reader as exercises.

Lemma 3 For $s, p \in \mathbb{N}$, we have

$$
\begin{aligned}
& \sum_{\nu=0}^{p}\left(\begin{array}{l}
p \\
v
\end{array}\right) \frac{\beta_{\nu}^{(k)}}{p-v+2}=\left(\begin{array}{c}
p+1 \\
s
\end{array}\right) \frac{\beta_{p-s+1}^{(k)}(1)}{p+1}+\frac{s-1}{p+1}\left(\begin{array}{c}
p+2 \\
s
\end{array}\right) \frac{\beta_{p-s+2}^{(k)}(1)}{p+2}, \\
& \sum_{\nu=0}^{p-s+1}\left(\begin{array}{l}
p \\
v
\end{array}\right)\left(\begin{array}{c}
p-v+2 \\
s
\end{array}\right) \frac{\beta_{v}^{(k)}}{p-v+2} \\
& =\left(\begin{array}{c}
p+1 \\
s
\end{array}\right) \frac{\beta_{p-s+1}^{(k)}(1)}{p+1}+\frac{s-1}{p+1}\left(\begin{array}{c}
p+2 \\
s
\end{array}\right) \frac{\beta_{p-s+2}^{(k)}(1)}{p+2}-\frac{1}{s}\left(\begin{array}{c}
p \\
s-2
\end{array}\right) \beta_{p-s+2}^{(k)},
\end{aligned}
$$

and

$$
\sum_{s=0}^{p}\left(\begin{array}{l}
p \\
s
\end{array}\right) \beta_{s}^{(k)} \frac{1}{p+2-s}=\frac{\beta_{p+1}^{(k)}(1)}{p+1}-\frac{\beta_{p+2}^{(k)}(1)}{(p+1)(p+2)}+\frac{\beta_{p+2}^{(k)}}{(p+1)(p+2)}
$$


As a further generalization of Apostol's Dedekind sums, we study poly-Dedekind sums associated with poly-Bernoulli functions of index $k$, which are given by

$$
T_{p}^{(k)}(h, m)=\sum_{\mu=1}^{m-1} \frac{\mu}{m} \bar{\beta}_{p}^{(k)}\left(\frac{h \mu}{m}\right)
$$

where $h, m, p \in \mathbb{N}, k \in \mathbb{Z}$, and $\bar{\beta}_{p}^{(k)}(x)=\beta_{p}^{(k)}(\langle x\rangle)$ are the poly-Bernoulli functions of index $k$.

Note that

$$
T_{p}^{(1)}(h, m)=\sum_{\mu=1}^{m-1} \frac{\mu}{m} \bar{B}_{p}\left(\frac{h \mu}{m}\right)=S_{p}(h, m) .
$$

The two identities in Lemma 4 can be proved in the same way as in Proposition 6 and Theorem 7 in [13], while the identity in Lemma 5 can be shown just as in Theorem 8 in [13]. Therefore their proofs are left to the reader.

Lemma 4 Let $p$ be an odd positive integer $\geq 3$, and $m \in \mathbb{N}$. Then we have

$$
\begin{aligned}
m^{p} & T_{p}^{(k)}(1, m) \\
& =\sum_{\nu=0}^{p}\left(\begin{array}{l}
p \\
v
\end{array}\right) \frac{\beta_{\nu}^{(k)}}{p+2-v} m^{p+1}+\sum_{i=1}^{p-1} \sum_{\nu=0}^{p+1-i}\left(\begin{array}{l}
p \\
v
\end{array}\right)\left(\begin{array}{c}
p+2-v \\
i
\end{array}\right) \frac{\beta_{v}^{(k)}}{p+2-v} B_{i} m^{p+1-i}+B_{p+1}
\end{aligned}
$$

and

$$
\begin{aligned}
(p+1) m^{p} T_{p}^{(k)}(1, m) & \\
= & \sum_{i=0}^{p+1}\left(\begin{array}{c}
p+1 \\
i
\end{array}\right) B_{i} m^{p+1-i} \beta_{p+1-i}^{(k)}(1) \\
& +\frac{1}{p+2} \sum_{i=0}^{p+1}\left(\begin{array}{c}
p+2 \\
i
\end{array}\right)(i-1) B_{i} m^{p+1-i}\left(\beta_{p+2-i}^{(k)}(1)-\beta_{p+2-i}^{(k)}\right)
\end{aligned}
$$

Lemma 5 For $m, n, h \in \mathbb{N}$ with $(h, m)=1$, and $p$ any positive odd integer $\geq 3$, we have

$$
\begin{aligned}
& \sum_{s=0}^{p+1}\left(\begin{array}{c}
p+1 \\
s
\end{array}\right) B_{s} \beta_{p+1-s}^{(k)}(1)(m h)^{p+1-s} \\
& \quad=m^{p} \sum_{\mu=0}^{m-1} \sum_{s=0}^{p+1}\left(\begin{array}{c}
p+1 \\
s
\end{array}\right) h^{s} \beta_{s}^{(k)}\left(\frac{\mu}{m}\right) B_{p+1-s}\left(h-\left[\frac{h \mu}{m}\right]\right) .
\end{aligned}
$$

For $d \in \mathbb{N}$, we observe that

$$
\begin{aligned}
\sum_{n=0}^{\infty} \beta_{n}^{(k)}(x) \frac{t^{n}}{n !} & =\frac{\operatorname{Li}_{k}\left(1-e^{-t}\right)}{e^{t}-1} e^{x t}=\frac{\operatorname{Li}_{k}\left(1-e^{-t}\right)}{e^{d t}-1} \sum_{i=0}^{d-1} e^{(i+x) t} \\
& =\frac{1}{d t} \operatorname{Li}_{k}\left(1-e^{-t}\right) \sum_{i=0}^{d-1} \frac{d t}{e^{d t}-1} e^{\left(\frac{i+x}{d}\right) d t}
\end{aligned}
$$




$$
\begin{aligned}
& =\sum_{j=0}^{\infty} d^{j-1} \sum_{i=0}^{d-1} B_{j}\left(\frac{x+i}{d}\right) \frac{t^{j}}{j !} \frac{1}{t} \sum_{l=1}^{\infty} \frac{l !}{l^{k}} \frac{1}{l !}\left(1-e^{-t}\right)^{l} \\
& =\sum_{j=0}^{\infty} d^{j-1} \sum_{i=0}^{d-1} B_{j}\left(\frac{x+i}{d}\right) \frac{t^{j}}{j !} \frac{1}{t} \sum_{l=1}^{\infty} \frac{(-1)^{l} l !}{l^{k}} \sum_{m=l}^{\infty} S_{2}(m, l) \frac{(-t)^{m}}{m !} \\
& =\sum_{j=0}^{\infty} d^{j-1} \sum_{i=0}^{d-1} B_{j}\left(\frac{x+i}{d}\right) \frac{t^{j}}{j !} \sum_{m=0}^{\infty} \frac{1}{m+1} \sum_{l=1}^{m+1} \frac{l !(-1)^{l+m-1}}{l^{k}} S_{2}(m+1, l) \frac{t^{m}}{m !} \\
& =\sum_{n=0}^{\infty}\left(\sum_{j=0}^{n} \sum_{i=0}^{d-1} \sum_{l=1}^{n-j+1}\left(\begin{array}{l}
n \\
j
\end{array}\right) d^{j-1} B_{j}\left(\frac{x+i}{d}\right) \frac{l !(-1)^{n-j+1-l}}{(n-j+1) l^{k}} S_{2}(n-j+1, l)\right) \frac{t^{n}}{n !} .
\end{aligned}
$$

Therefore, by (19), we obtain the following theorem.

Theorem 6 For $k \in \mathbb{Z}, d \in \mathbb{N}$, and $n \geq 0$, we have

$$
\beta_{n}^{(k)}(x)=\sum_{j=0}^{n} \sum_{i=0}^{d-1} \sum_{l=1}^{n-j+1}\left(\begin{array}{l}
n \\
j
\end{array}\right) d^{j-1} B_{j}\left(\frac{x+i}{d}\right) \frac{l !(-1)^{n-j+1-l}}{(n-j+1) l^{k}} S_{2}(n-j+1, l) .
$$

By (18), Lemmas 3-5, and Theorem 6, we get

$$
\begin{aligned}
& h m^{p} T_{p}^{(k)}(h, m)+m h^{p} T_{p}^{(k)}(m, h) \\
& =h m^{p} \sum_{\mu=0}^{m-1} \frac{\mu}{m} \bar{\beta}_{p}^{(k)}\left(\frac{h \mu}{m}\right)+m h^{p} \sum_{\nu=0}^{h-1}\left(\frac{\mu}{h}\right) \bar{\beta}_{p}^{(k)}\left(\frac{m v}{h}\right) \\
& =h m^{p} \sum_{\mu=0}^{m-1} \frac{\mu}{m} \sum_{j=0}^{p} h^{j-1}\left(\begin{array}{l}
p \\
j
\end{array}\right) \sum_{\nu=0}^{h-1} \sum_{l=1}^{p-j+1} \frac{l !(-1)^{p-j+1-l}}{(p-j+1) l^{k}} S_{2}(p-j+1, l) \bar{B}_{j}\left(\frac{\mu}{m}+\frac{v}{h}\right) \\
& +m h^{p} \sum_{\nu=0}^{h-1} \frac{v}{h} \sum_{j=0}^{p} m^{j-1}\left(\begin{array}{l}
p \\
j
\end{array}\right) \sum_{\mu=0}^{m-1} \sum_{l=1}^{p-j+1} \frac{l !(-1)^{p-j+1-l}}{(p-j+1) l^{k}} S_{2}(p-j+1, l) \bar{B}_{j}\left(\frac{v}{h}+\frac{\mu}{m}\right) \\
& =\sum_{\mu=0}^{m-1} \frac{\mu}{m} \sum_{j=0}^{p} m^{p-j}(m h)^{j}\left(\begin{array}{l}
p \\
j
\end{array}\right) \sum_{\nu=0}^{h-1} \sum_{l=1}^{p-j+1} \bar{B}_{j}\left(\frac{\mu}{m}+\frac{v}{h}\right) \frac{l ! S_{2}(p-j+1, l)}{(p-j+1) l^{k}}(-1)^{p-j+1-l} \\
& +\sum_{\nu=0}^{h-1} \frac{v}{h} \sum_{j=0}^{p} h^{p-j}(m h)^{j}\left(\begin{array}{l}
p \\
j
\end{array}\right) \sum_{\mu=0}^{m-1} \sum_{l=1}^{p-j+1} \bar{B}_{j}\left(\frac{v}{h}+\frac{\mu}{m}\right) \frac{l ! S_{2}(p-j+1, l)}{(p-j+1) l^{k}}(-1)^{p-j+1-l} \\
& =\sum_{\mu=0}^{m-1} \sum_{j=0}^{p} \sum_{\nu=0}^{h-1} \sum_{l=1}^{p-j+1}(\mu h)(m h)^{-1} m^{p-j}(m h)^{j}\left(\begin{array}{l}
p \\
j
\end{array}\right) \\
& \times \bar{B}_{j}\left(\frac{\mu}{m}+\frac{v}{h}\right) \frac{l ! S_{2}(p-j+1, l)}{(p-j+1) l^{k}}(-1)^{p-j+1-l} \\
& +\sum_{\mu=0}^{m-1} \sum_{j=0}^{p} \sum_{\nu=0}^{h-1} \sum_{l=1}^{p-j+1}(m v)(m h)^{-1} h^{p-j}(m h)^{j}\left(\begin{array}{l}
p \\
j
\end{array}\right) \\
& \times \bar{B}_{j}\left(\frac{v}{h}+\frac{\mu}{m}\right) \frac{l ! S_{2}(p-j+1, l)}{(p-j+1) l^{k}}(-1)^{p-j+1-l}
\end{aligned}
$$




$$
\begin{aligned}
= & \sum_{\mu=0}^{m-1} \sum_{j=0}^{p} \sum_{\nu=0}^{h-1} \sum_{l=1}^{p-j+1} \frac{(m h)^{j-1} l ! S_{2}(p-j+1, l)}{(p-j+1) l^{k}} \\
& \times\left(\begin{array}{c}
p \\
j
\end{array}\right)(-1)^{p-j+1-l}\left((\mu h) m^{p-j}+(m \nu) h^{p-j}\right) \bar{B}_{j}\left(\frac{v}{h}+\frac{\mu}{m}\right) .
\end{aligned}
$$

Therefore, by (20), we obtain the following reciprocity theorem for the poly-Dedekind sums associated with poly-Bernoulli functions with index $k$.

Theorem 7 For $m, h, p \in \mathbb{N}$ and $k \in \mathbb{Z}$, we have

$$
\begin{aligned}
h m^{p} & T_{p}^{(k)}(h, m)+m h^{p} T_{p}^{(k)}(m, h) \\
= & \sum_{\mu=0}^{m-1} \sum_{j=0}^{p} \sum_{\nu=0}^{h-1} \sum_{l=1}^{p-j+1} \frac{(m h)^{j-1} l ! S_{2}(p-j+1, l)}{(p-j+1) l^{k}} \\
& \times\left(\begin{array}{c}
p \\
j
\end{array}\right)(-1)^{p-j+1-l}\left((\mu h) m^{p-j}+(m v) h^{p-j}\right) \bar{B}_{j}\left(\frac{v}{h}+\frac{\mu}{m}\right) .
\end{aligned}
$$

In case of $k=1$, by making use of Corollary 2, we obtain the following reciprocity relation for the generalized Dedekind sums defined by Apostol.

Corollary 8 For $m, h, p \in \mathbb{N}$, we have

$$
\begin{aligned}
h m^{p} T_{p}^{(1)}(h, m)+m h^{p} T_{p}^{(1)}(m, h) & =m h^{p} S_{p}(h, m)+m h^{p} S_{p}(m, h) \\
& =\sum_{\mu=0}^{m-1} \sum_{\nu=0}^{h-1}(m h)^{p-1}(\mu h+m \nu) \bar{B}_{p}\left(\frac{v}{h}+\frac{\mu}{m}\right) .
\end{aligned}
$$

\section{Conclusion}

The quantity called the Dedekind sum,

$$
S(h, m)=\sum_{\mu=1}^{m-1} \frac{\mu}{m} \bar{B}_{1}\left(\frac{h \mu}{m}\right),
$$

occurs in the transformation behavior of the logarithm of the Dedekind eta-function under substitutions from the modular group. It was shown by Dedekind that they satisfy the following reciprocity relation:

$$
S(h, m)+S(m, h)=\frac{1}{12}\left(\frac{h}{m}+\frac{1}{h m}+\frac{m}{h}\right)-\frac{1}{4}
$$

if $h$ and $m$ are relatively prime positive integers.

Apostol considered the generalized Dedekind sums

$$
S_{p}(h, m)=\sum_{\mu=1}^{m-1} \frac{\mu}{m} \bar{B}_{p}\left(\frac{h \mu}{m}\right)
$$


and derived a reciprocity relation for them. Recently, as one generalization of the generalized Dedekind sums, the poly-Dedekind sums

$$
S_{P}^{(k)}(h, m)=\sum_{\mu=1}^{m-1} \frac{\mu}{m} \bar{B}_{p}^{(k)}\left(\frac{h \mu}{m}\right),
$$

associated with the type 2 poly-Bernoulli functions of arbitrary indices, were introduced and were shown to satisfy a reciprocity relation. In this paper, as another generalization of the generalized Dedekind sums, we considered the poly-Dedekind sums

$$
T_{p}^{(k)}(h, m)=\sum_{\mu=1}^{m-1} \frac{\mu}{m} \bar{\beta}_{p}^{(k)}\left(\frac{h \mu}{m}\right)
$$

associated with the poly-Bernoulli functions of arbitrary indices, and derived a reciprocity relation for these poly-Dedekind sums.

\section{Acknowledgements}

The authors would like to thank the reviewers for their suggestions that helped improve the original manuscript in its present form. Also, the authors thank Jangjeon Research Institute for Mathematical Sciences for the support of this research.

\section{Funding}

This research was funded by the National Natural Science Foundation of China (No. 11871317, 11926325, 11926321).

Availability of data and materials

Not applicable.

\section{Ethics approval and consent to participate}

All authors reveal that there is no ethical problem in the production of this paper.

Competing interests

The authors declare that they have no competing interests.

Consent for publication

All authors want to publish this paper in this journal.

\section{Authors' contributions}

All authors contributed equally to the manuscript and typed, read, and approved the final manuscript.

\section{Author details}

${ }^{1}$ School of Science, Xi'an Technological University, Xi'an, 710021, Shaanxi, P.R. China. ${ }^{2}$ Department of Mathematics, Sogang University, Seoul 121-742, Republic of Korea. ${ }^{3}$ Department of Mathematics, Kwangwoon University, Seoul 139-701, Republic of Korea.

\section{Publisher's Note}

Springer Nature remains neutral with regard to jurisdictional claims in published maps and institutional affiliations.

Received: 25 September 2020 Accepted: 11 November 2020 Published online: 23 November 2020

\section{References}

1. Apostol, T.M.: Generalized Dedekind sums and transformation formulae of certain Lambert series. Duke Math. J. 17, 147-157 (1950)

2. Apostol, T.M.: Theorems on generalized Dedekind sums. Pac. J. Math. 2, 1-9 (1952)

3. Can, M., Cenkci, M., Kurt, V., Simsek, Y.: Twisted Dedekind type sums associated with Barnes' type multiple Frobenius-Euler l-functions. Adv. Stud. Contemp. Math. (Kyungshang) 18(2), 135-160 (2009)

4. Carlitz, L.: A reciprocity theorem and a three-term relation for generalized Dedekind-Rademacher sums. Acta Arith. 37, 117-132 (1980)

5. Carlitz, L.: An application of the reciprocity theorem for Dedekind sums. Fibonacci Q. 22(3), 266-270 (1984)

6. Kaneko, M.: Poly-Bernoulli numbers. J. Théor. Nr. Bordx. 9(1), 221-228 (1997)

7. Kim, D.S., Kim, T.: A note on polyexponential and unipoly functions. Russ. J. Math. Phys. 26(1), 40-49 (2019)

8. Kim, D.S., Kim, T.: A note on a new type of degenerate Bernoulli numbers. Russ. J. Math. Phys. 27(2), $227-235$ (2020) 
9. Kim, T:: A note on $p$-adic q-Dedekind sums. C. R. Acad. Bulgare Sci. 54(10), 37-42 (2001)

10. Kim, T.: Note on Dedekind type DC sums. Adv. Stud. Contemp. Math. (Kyungshang) 18(2), 249-260 (2009)

11. Kim, T.: Note on q-Dedekind-type sums related to q-Euler polynomials. Glasg. Math. J. 54(1), 121-125 (2012)

12. Kim, T., Kim, D.S., Kim, H.-Y., Lee, H., Jang, L.-C.: Degenerate poly-Bernoulli polynomials arising from degenerate polylogarithm. Adv. Differ. Equ. 2020, 444 (2020)

13. Kim, T., Kim, D.S., Lee, H., Jang, L.-C.: Identities on poly-Dedekind sums. Adv. Differ. Equ. 2020, 563 (2020)

14. Milovanović, G.V., Simsek, Y.: Dedekind and Hardy type sums and trigonometric sums induced by quadrature formulas. In: Raigorodskii, A., Rassias, M. (eds.) Trigonometric Sums and Their Applications, pp. 183-228. Springer, Cham (2020) https://doi-org-443.webvpn.fjmu.edu.cn/10.1007/978-3-030-37904-9_10

15. Simsek, Y: Relations between theta-functions Hardy sums Eisenstein and Lambert series in the transformation formula of $\log \eta_{a, h}(z)$. J. Number Theory 99, 338-360 (2003)

16. Simsek, Y.: Generalized Dedekind sums associated with the Abel sum and the Eisenstein and Lambert series. Adv. Stud. Contemp. Math. (Kyungshang) 9(2), 125-137 (2004)

17. Simsek, Y.: Remarks on reciprocity laws of the Dedekind and Hardy sums. Adv. Stud. Contemp. Math. (Kyungshang) 12(2), 237-246 (2006)

18. Simsek, Y.: q-Dedekind type sums related to q-zeta function and basic L-series. J. Math. Anal. Appl. 318(1), 333-351 (2006)

19. Simsek, Y.: Special functions related to Dedekind-type DC-sums and their applications. Russ. J. Math. Phys. 17(4), 495-508 (2010)

20. Simsek, Y., Acikgoz, M.: Remarks on Dedekind eta function, theta functions and Eisenstein series under the Hecke operators. Adv. Stud. Contemp. Math. (Kyungshang) 10(1), 15-24 (2005)

21. Simsek, Y., Yang, S.: Transformation of four Titchmarsh-type infinite integrals and generalized Dedekind sums associated with Lambert series. Adv. Stud. Contemp. Math. (Kyungshang) 9(2), 195-202 (2004)

\section{Submit your manuscript to a SpringerOpen ${ }^{\circ}$ journal and benefit from:}

- Convenient online submission

- Rigorous peer review

- Open access: articles freely available online

- High visibility within the field

\section{- Retaining the copyright to your article}

